\title{
Correlation of Predicted Birth Weight at 20-24 Weeks of Gestational Age using the Formula devised by Chi-Feng Su to Actual Birth Weight
}

\author{
${ }^{1}$ Bandi H Reddy, ${ }^{2} \mathrm{~N}$ Nagendra Prasad, ${ }^{3}$ Rangaiah Nagaratnamma, ${ }^{4}$ MoOrkoth PA Sailakshmi
}

\begin{abstract}
Aim: The aim of the study is to use the formula demonstrated by Chi-Feng Su to determine the estimated birth weight (EBW) at 20-24 weeks gestation and try to find out the correlation between the estimated fetal weight with the actual birth weight in our local population.
\end{abstract}

Materials and methods: It is a retrospective study done at the Department of Obstetrics and Gynecology, RajaRajeswari Medical College, Bengaluru. Fifty patients who were booked, followed up, and delivered at our institution were selected for our study after meeting inclusion and exclusion criteria. Participant's demographic characters like age, gravida, weight, body mass index (BMI), last menstrual period (LMP), and others were noted down as per the protocol. Their mid-pregnancy BMI, and fetal biometry report at 20-24 weeks were noted. Using the study formula, their anticipated fetal weight was calculated. After delivery, the exact birth weight was noted and was correlated with the predicted weight.

Results: In the study, majority (66\%) aged less than 25 years and only 3 patients were aged above 30 years; $50 \%$ of the cases were second gravida; $60 \%$ of the participants had BMI between 20 and $25,16 \%$ has $\mathrm{BMI}$ above 25 , and $24 \%$ has $\mathrm{BMI}$ less than 20. The best correlation was seen in the weight group between 2.6 and $3.5 \mathrm{~kg}$.

Conclusion: The formula demonstrated by Chi-Feng Su had variance as high as 0.303 . Our study also showed high variability when the error was $+17.6 \%$ with birth weight $>2.5 \mathrm{~kg}$.

Keywords: Actual birth weight, Body mass index, Estimated fetal weight.

How to cite this article: Reddy BH, Prasad NN, Nagaratnamma R, Sailakshmi MPA. Correlation of Predicted Birth Weight at 20-24 Weeks of Gestational Age using the Formula devised by Chi-Feng Su to Actual Birth Weight. J Med Sci 2018; 4(2):35-37.

Source of support: Nil

Conflict of interest: None

\footnotetext{
${ }^{1}$ Postgraduate Student, ${ }^{2,3}$ Professor, ${ }^{4}$ Professor and Head

${ }^{1-4}$ Department of Obstetrics and Gynecology, RajaRajeswari Medical College \& Hospital, Bengaluru, Karnataka, India

Corresponding Author: Bandi H Reddy, Postgraduate Student Department of Obstetrics and Gynecology, RajaRajeswari Medical College \& Hospital, Bengaluru, Karnataka, India, e-mail: drnnprasad@gmail.com
}

\section{INTRODUCTION}

Determining fetal weight accurately prior to delivery is very essential and is useful for taking clinical decision in labor, thereby improving perinatal outcome.

The estimation of fetal weight has undergone tremendous transformation from clinical estimation to present-day assessment by multiple parameters in ultrasonography. Clinical estimation of fetal weight relied on abdominal examination findings like symphysis fundal height (SFH), abdominal circumference (AC), and other parameters. Various clinical formulas like Johnson's and Dawn's are used for the estimation of fetal weight near term.

In the past decades, improvement in ultrasound imaging and better availability of equipment, has made possible widespread use of ultrasound for assessing fetal weight across all gestational ages. Various formulas used, such as Hadlock's, Shepard's, Campbell's, etc., predict the fetal weight with a reasonable error of $6-15 \%{ }^{1}$

All the above methods measure the estimated fetal weight near term, but we have very few methods to predict the birth weight at late second trimester or early third trimester. There are few studies which state fetus with intrauterine growth restriction (IUGR), and the biometry values deviate from the normogram from the second trimester itself. However, there are no reliable methods or formulas to predict IUGR.

Intrauterine growth restriction is defined as the pathologic inhibition of intrauterine fetal growth and the failure of the fetus to achieve its growth potential. ${ }^{2}$ The IUGR is seen in about $10 \%$ of pregnancies. ${ }^{3}$ Fetal growth is known to be as important predictor of pregnancy outcome as IUGR is associated with increased fetal and neonatal morbidity and mortality. The IUGR is also linked to neonatal complications, such as prematurity, cerebral palsy, intrauterine device (IUD), and neonatal deaths. ${ }^{4}$

There have been a few reports about the studies to predict the birth weight at term as early as midpregnancy. ${ }^{5-7}$ Chi-Feng Su et al, ${ }^{8}$ published a study which used a unique formula to predict birth weight using fetal biometry and maternal characteristics, as early as $20-24$ weeks gestation. 
They considered maternal weight factors, pregnancy time interval, and sonographic fetal biometry, and developed a simple and easy mathematical equation for term birth weight estimation at mid-pregnancy. The formula is

$\mathrm{EBW}=-700+49.766 \times($ mid-pregnancy BMI $)+13.362 \times$ (time interval from mid-pregnancy to term delivery in days) + $68.696 \times$ (abdominal circumference in $\mathrm{cm})$.

\section{AIM}

The aim of the study is to use the formula proposed by Chi-Feng Su et al and try to find out the correlation between the estimated fetal weight with the actual birth weight in our local population.

\section{MATERIALS AND METHODS}

It is a retrospective study done at the Department of Obstetrics and Gynecology, RajaRajeswari Medical College, Bengaluru, Karnataka, India. Fifty patients who were booked, followed up, and delivered at our institution were selected for our study after meeting inclusion and exclusion criteria.

\section{Inclusion Criteria}

- Singleton pregnancy

\section{Exclusion Criteria}

- Multiple pregnancy.

- Obstetrics complications like pregnancy-induced hypertension, gestational diabetes mellitus

- Medical disorders like congenital heart diseases.

Participant's demographic characters like age, gravida, weight, BMI, LMP, and others were noted down as per the protocol. Their mid-pregnancy BMI, fetal biometry report at 20 to 24 weeks were noted. Using the study formula, their anticipated fetal weight was calculated. After delivery, the exact birth weight was noted and was correlated with the predicted weight.

\section{RESULTS}

Out of 50 participants analyzed in the study, majority $(66 \%)$ were aged less than 25 years and only 3 patients were aged above 30 years; $50 \%$ of the cases were second gravida; $60 \%$ of the participants had BMI between 20 and $25,16 \%$ had BMI above 25, and 24\% had BMI less than 20 (Table 1).

Table 2 shows the correlation of EBW to the actual birth weight. According to the formula, there were no babies with anticipated birth weight of $<2 \mathrm{~kg}$, but there were 9 babies with actual birth weight of $<2 \mathrm{~kg}$. The best correlation was seen in the weight group between 2.6 and $3.5 \mathrm{~kg}$. The estimated weight in this group showed
Table 1: Demography

\begin{tabular}{lcl}
\hline Age (years) & $<25$ & 33 \\
& $25-30$ & 14 \\
Gravida & $>30$ & 3 \\
& I & 9 \\
& II & 25 \\
BMI & $>$ II & 16 \\
& $<20$ & 12 \\
& $20-25$ & 30 \\
& $>25$ & 8 \\
\hline
\end{tabular}

Table 2: Correlation of EBW to actual birth weight

\begin{tabular}{cll}
\hline Range & Estimated weight $(\mathrm{kg})$ & Actual weight $(\mathrm{kg})$ \\
\hline$<2.0$ & 0 & 9 \\
$2.1-2.5$ & 10 & 5 \\
$2.6-3.0$ & 5 & 22 \\
$3.1-3.5$ & 29 & 11 \\
$>3.5$ & 6 & 3 \\
\hline
\end{tabular}

34 babies, and 33 babies were born with actual birth weight ranging between 2.6 and $3.5 \mathrm{~kg}$. In the weight group $>3.5 \mathrm{~kg}$, there were 6 cases in the estimated group and 3 cases in the actual birth weight group showing reasonable correlation.

\section{DISCUSSION}

Various formulas or methods are used to estimate the fetal weight during antenatal care, but actual birth weight can only be measured after child birth. Chien et $\mathrm{al}^{9}$ used ultrasound measurement of various fetal biometric parameters at term to assess fetal weight and found reasonable correlation with actual birth weight. They stated that the mean error and the rate of estimated weight was within $10 \%$ of the birth weight.

However, predicting macrosomia or IUGR at midtrimester was not possible with their formula.

We have used the formula that was constructed by Chi-Feng Su et al as a predictive model that could be used during mid-pregnancy in order to predict the birth weight.

The formula was EBW $=-700+49.766 \times$ (mid-pregnancy $\mathrm{BMI})+13.362 \times$ (time interval from mid-pregnancy to term delivery [days] $)+68.696 \times(\mathrm{AC})$. The formula was used to test the possibility of predicting small or large babies at 20 and 24 weeks of gestation. This formula would be valuable for obstetricians to provide appropriate prenatal care and to prevent the birth of very small or very large babies.

In their study, many contributory factors were included related to birth weight, such as gestational age, maternal characteristics, and fetal ultrasound biometrics. Maternal weight variables during pregnancy were significant factors that affect the newborn birth weight. 
In their study, maternal weight gain and BMI gain had a significant correlation $(r=0.18, p<0.05)$. However, maternal BMI and weight at 20-24 weeks of gestation showed the highest correlation coefficient $(\mathrm{r}=0.388$ and 0.358 respectively, $\mathrm{p}<0.05)$.

Our study shows that the formula was reliable with an error of $\pm 2.9 \%$ in predicting the weight among the group of $2.6-3.5 \mathrm{~kg}$. Among the weight group $>3.5 \mathrm{~kg}$, 6 participants were predicted but actual weight of $>3.5 \mathrm{~kg}$ was seen in 3 patients.

However, most problem group was $<2 \mathrm{~kg}$, where no participant had estimated weight $<2 \mathrm{~kg}$, and actual weight was $<2 \mathrm{~kg}$ in 9 participants. In the actual weight group, there were 14 participants with weight less than $2.5 \mathrm{~kg}$ with an average weight of $2.21 \mathrm{~kg}$. But the average EBW of these 14 participants was $2.605 \mathrm{~kg}$ with an error percent of $+17.6 \%$.

Similar to the original study, the average error in the estimation of fetal weight when all the 50 participants were considered was $\pm 0.333 \mathrm{~kg}$.

\section{CONCLUSION}

The formula demonstrated by Chi-Feng Su had variance as high as 0.303 . Our study also showed high variability when the error was $+17.6 \%$ with birth weight $>2 \mathrm{~kg}$.

However, predicting term birth weight at midpregnancy had some limitations. There are many unpredictable factors that affect birth weight during the period from mid-pregnancy to term delivery, and some factors, such as maternal behavior, nutrition, and exercise vary individually. As much as $30 \%$ of the factors for preventing larger and smaller infants at delivery can be predicted as early as 20-24 weeks of gestation; therefore, it would be worthwhile to plan necessary interventions to prevent neonatal morbidity and mortality.

\section{REFERENCES}

1. Kurmanavicius J, Burkhardt T, Wisser J, Huch R. Ultrasonographic fetal weight estimation: accuracy of formulas and accuracy of examiners by birth weight from 500 to 5000 g. J Perinat Med 2004;32(2):155-161.

2. ACOG Practice bulletin no. 134: fetal growth restriction. Obstet gynecol 2013 May;121(5):1122-1133.

3. UnterscheiderJ,DalyS, Geary MP,Kennelly MM,McAuliffeFM, O'Donoghue K, Hunter A, Morrison JJ, Burke G, Dicker P, et al. Optimizing the definition of intrauterine growth restriction: the multicenter prospective PORTO study. Am J Obstet Gynecol 2013 Apr;208(4):290e.1-290e.6.

4. Anderson NH, Sadler LC, Stewart AW, McCowan LM. Maternal and pathological pregnancy characteristics in customised birth weight centiles and identification of at-risk small-for-gestational-age infants: a retrospective cohort study. BJOG 2012 Jun;119(7):848-856.

5. Ben-Haroush A, Yogev Y, Hod M. Predictive value of a single early fetal weight estimate in normal pregnancies. Eur J Obstet Gynecol Reprod Biol 2007 Feb;130(2):187-192.

6. Yoshida SH, Unno N, Kagawa H, Shinozuka N, Kozuma S, Taketani Y. Sonographic determination of fetal size from 20 weeks of gestation onward correlates with birth weight. J Obstet Gynecol Res 2001 Aug;27(4):205-211.

7. Honarvar M, Allahhyari M, Dehbashi S. Assessment of fetal weight based on ultrasonic femur length after the second trimester. Int J Gynaecol Obstet 2001 Apr;73(1):15-20.

8. Su CF, Tsai HJ, Lin CY, Ying TH, Wang PH, Chen GD. Prediction of newborn birth weight based on the estimation at 20-24 weeks of gestation. Taiwan J Obstet Gynecol 2010 Sep;49(3):285-290.

9. Chien PF, Owen $\mathrm{P}$, Khan KS. Validity of ultrasound estimation of fetal weight. Obstet Gynecol 2000 Jun;95(6 Pt 1):856-860. 